\title{
Die Schweinegrippe und die vermeintliche Gefährlichkeit von Virenmutationen
}

\author{
Eine Studie über die Rolle des Wirts als Determinante gefährlicher Pandemien
}

\author{
Mac Heynar Ramos F. Johannes G. Schmidt \\ Stiftung Paracelsus heute, Einsiedeln, Schweiz
}

\author{
Schlüsselwörter \\ Schweinegrippe · Wirt und Krankheit · Virus • Impfung · Gesamteffekt . \\ Scheinerfolg
}

\section{Zusammenfassung}

Wir wollen die Frage studieren, ob die Idee gefährlicher Virusmutationen gut begründet ist oder ob nicht alternative Erklärungen plausibler sind. Wird das Wechselspiel zwischen Krankheit und Wirt in die Betrachtungen einbezogen, dann bestimmt nicht das Virus allein einen fatalen Verlauf, sondern ebenso die Widerstandskräfte des Wirts. So gesehen war die Spanische Grippe das Ergebnis der prekären Verhältnisse am Ende des 1. Weltkrieges und nicht einfach das Ergebnis einer gefährlichen Virus-Mutante. Immer wieder und auch bei der Schweinegrippe zeigt sich dieses Wirts-Muster: Geschwächte werden ernsthaft krank und können sterben, bei ordentlichen Widerstands- und Gesunderhaltungskräften erfolgen hingegen nur leichte Krankheitserscheinungen. Ein Wirt mit einer guten Abwehr kommt ohne Impfungen gut durch und hat auch keine Impfnebenwirkungen zu fürchten. Ein Wirt mit einer prekären oder momentan geschwächten Abwehrkraft kann durch Viren, aber - in einem ebenso ungewissen Ausmass - auch durch Impfungen beeinträchtigt werden. Nicht die spezifische Immunität und der entsprechende Impfschutz, sondern «Mutationen» (bzw. Unterschiede) in der unspezifischen Immunbereitschaft des Wirts korrelieren mit einem komplizierten Ausgang von Virusinfektionen. Es ist interessant, darauf hinzuweisen, dass der Wirtszustand auch bei anderen Krankheiten - z.B. Krebs, Rheuma, Multiple Sklerose usw. - die entscheidende und wissenschaftlich vernachlässigte Determinante von Krankheitsverläufen und Mortalität sein könnte und nicht die scheinbare Aggressivität oder Bösartigkeit der jeweiligen Krankheit.

\section{Keywords}

Swine flu $\cdot$ Host and disease - Virus · Vaccination · Total effect . Pseudo success

\section{Summary}

Swine Flu: Is Virus Mutation Dangerous? A Study on the Importance of the Host as a Determinant of a Dangerous Pandemic

We will study the question to what extent dangerous virus mutations really exist or whether this is a potential myth which has ignored other plausible explanations. The interplay between host and disease is an important medical issue which nowadays is often ignored. The Spanish flu was likely the result only of the precarious conditions at the end of World War I which have compromised host resistance. Other viruses and H1N1, repeatedly, show the same pattern: A weak host gets sick and may even die; if host resistance is in order, virus infection shows an innocuous course. A competent host with a proper resistance does very well without vaccination and also does not need to fear adverse effects of vaccination. A host with a weakened defence, on the other hand, can be affected by viruses as well as by vaccinations - to an extent similarly unknown in both cases. It is not the specific immunity, but 'mutations' (or changes) in the non-specific host resistance that correlate with a complicated outcome of viral infections. It is interesting to note that the host condition for other diseases - e.g. cancer, rheumatism, multiple sclerosis etc. - may also be the crucial and scientifically neglected determinant of disease progression and mortality, not the alleged virulence or malignancy of the specific disease.

$\begin{array}{ll}\text { KARGER } & \text { @ 2010 S. Karger GmbH, Freiburg } \\ \text { Fax }+497614520714 & \text { Accessible online at: } \\ \begin{array}{l}\text { Information@Karger.de } \\ \text { www.karger.com }\end{array} & \text { www.karger.com/szg }\end{array}$

Dr. med. Johannes G. Schmidt Stiftung Paracelsus heute Praxiszentrum Meinradsberg Ilgenweidstrasse 3, 8840 Einsiedeln, Schweiz

Tel. +41 55-4188191, Fax -4188192 schmidt@paracelsus-heute.ch 


\section{Einführung}

Alle hatten Impfstoff gekauft - ausser Polen und wenige andere Länder. Die Pandemiewarnung der World Health Organization (WHO) zur Schweinegrippe (H1N1) und die entsprechenden Impfempfehlungen stehen aber in der Kritik [1]. Kritisiert wird die Manipulation der PandemieKriterien sowie eine zu grosse Nähe der WHO zur Pharmaindustrie, sodass sich die WHO dem Verdacht der Gefälligkeit zugunsten dieser Industrie aussetzt. Allerdings musste die WHO ihre Beschlüsse zum Schutz der Bevölkerung aus damaliger Sicht vornehmen, wobei die verbreitete Meinung im Raum stand, dass gefährliche Virenmutationen drohen könnten. Uns scheint deshalb, dass es sich um eine globale Fehlleistung über die WHO hinaus handelt, die durch eine kollektive Fehlwahrnehmung der Determinanten von Gesundheit zustande gekommen ist. Denn die globale «Medical Community» übte sich im scholastischen Vor- und Nachbeten dieser Gefährlichkeitshypothese, die sie von der Spanische Grippe von 1918 herleiten will $[2,3]$.

Wir wollen die Frage prüfen, ob die Idee gefährlicher Virusmutationen tatsächlich gut begründet und unwiderlegbar ist oder ob nicht alternative Erklärungen möglich und plausibler sind. Dabei ist die klinische Relevanz oder Gefährlichkeit von Virenmutationen nicht eine Frage virologischer Methoden, und sie lässt sich mit Erkenntnissen aus Laborstudien und Antigen-Analysen bzw. mit Antigen-Theorien nicht beantworten. Entscheidend für die Frage des klinischen Nutzens sind die klinischen Ergebnisse - epidemiologisch analysiert. Es braucht die Klinische Epidemiologie mit ihren systematischen Forschungsfragen, um eine zuverlässige Antwort über Wirksamkeit und Nutzen medizinischer Interventionen $\mathrm{zu}$ erarbeiten.

\section{Krankheit und Wirt}

Das Wechselspiel zwischen Krankheit und Wirt ist immer ein wichtiges medizinisches Thema $[4,5]$ gewesen, das in der modernen Medikalisierungseuphorie aber wenig Beachtung findet. Es ist leicht geworden, Risiko- und Krankheitsfaktoren - und Viren - als Bedrohung für die Gesundheit zu propagieren, ohne die Frage nach dem Wirt und seinen Widerstandskräften überhaupt noch zu stellen. Wir glauben, dass dies aus wissenschaftlicher Sicht falsch ist und die Medizin in die Irre geführt hat. Wo der (unspezifische) Wirtszustand bestimmt, ob eine Krankheit gutartig oder bösartig verläuft, dort sind «erfolgreiche» Manipulationen an (spezifischen) Krankheitsäusserungen ein Scheinerfolg. Wir müssen deshalb genau wissen, ob eine (nachhaltige) Verbesserung in Bezug auf die Gesamtmorbidität und -mortalität und auf das Leiden insgesamt vorliegt, bevor von Erfolg und Nutzen gesprochen werden kann.

So ist bekannt, dass die erste erfolgreiche Impfung überhaupt - die Pockenimpfung - zwar die häufige PockenKindersterblichkeit im Berlin der 1930er-Jahre praktisch zum Verschwinden gebracht hat, aber es ist weniger bekannt, dass die Kindersterblichkeit an allen Ursachen insgesamt unverändert blieb [6]. Denn letztlich konnte der anfällige Wirt konkurrierenden Mortalitätsursachen nicht trotzen, sodass die (unterernährten) Kinder insgesamt gleich häufig starben - ob gegen Pocken geimpft oder nicht. Thomas McKeown ist in seiner grossen epidemiologischen Studie der Frage nachgegangen, wie sich die Mortalität an Infektionskrankheiten zwischen 1860 und 1960 in England und Wales verändert hat, und hatte festgestellt, dass der Rückgang fast linear über die ganze Zeitspanne erfolgte, lange bevor medizinische Massnahmen getroffen werden konnten [7]. Die Einführung von Impfungen (und Antibiotika) brachten keine Veränderung in diesen säkularen Trend. McKeown glaubte, dass ausreichende Ernährung und bessere Wohnverhältnisse für die breite Bevölkerung seit Ende des 19. Jahrhunderts am besten mit dieser Beobachtung korrelierten.

Ein weiteres Beispiel sind die Masern, die bei uns noch bis in die 1970er-Jahre endemisch bei allen Kindern auftraten und praktisch nie zu Sterbefällen führten. In Drittweltländern waren und sind Masern hingegen oft einer der wichtigsten Kontributoren zur Kindersterblichkeit. Das (Masern-)Virus bestimmt also nicht einen fatalen Verlauf, sondern die geschwächten Widerstandskräfte des Wirts unter prekären Verhältnissen.

\section{Die Spanische Grippe}

Im Unterschied zu Masern und Pocken mutieren Influenzaviren, sodass sie keine generelle Immunität gegen Grippe hinterlassen. Die Spanische Grippe im Jahr 1918 gilt als der Paradefall einer gefährlichen Pandemie, welche durch eine gefährliche, neue Virenmutante ausgelöst worden sei, gegen die keine vorbestehende (spezifische) Immunität in der Bevölkerung bestanden habe. Mit ihr haben die WHO und die vielen nationalen Impfkommissionen ihr Vorgehen gegen die Schweinegrippe begründet und gerechtfertigt. In dieser Perzeption gänzlich ausgeblendet bleibt aber die damalige Situation des Wirts. Europa hatte 1918 einen verheerenden Krieg hinter sich. Bei der Spanischen Grippe stieg angesichts der vielen Todesfälle durch Grippe auch die Gesamtmortalität. Bei der ebenfalls gefürchteten Hongkong-Grippe 1957 und der Asiatischen Grippe 1968 zeigten sich hingegen keine Veränderungen in der Gesamtsterblichkeit [8].

Wenn man diese Ergebnisse ins rechte Licht rückt, dann war die Spanische Grippe wahrscheinlich nur das Ergebnis 
der prekären Verhältnisse am Ende des 1. Weltkriegs. Entbehrung und Schwächung auf der Seite des Wirts erklären ausreichend, weshalb das spanische Virus einen hohen Mortalitätszoll forderte, zum Teil auch unter jüngeren Menschen, denen die Kriegsfolgen und die Schwächung ihrer Abwehrkräfte nicht ohne Weiteres anzusehen waren. Man darf deshalb nicht einfach den Schluss ziehen, die spanische Virusmutante sei an sich gefährlich und besonders virulent gewesen.

\section{Die H1N1-Grippe}

In Übereinstimmung mit diesen epidemiologischen Beobachtungen zeigt sich auch bei der Schweinegrippe dieses Wirtsmuster (wie übrigens damals auch bei SARS (schweres akutes respiratorisches Syndrom)). Die dokumentierten Todesfälle zeigen in den weitaus meisten Fällen eine vorbestehende Morbidität beim Wirt [9]. Geschwächte werden ernsthaft krank und können sterben; bei ordentlichen Widerstands- und Gesunderhaltungskräften erfolgt hingegen nicht mehr als ein leichter Schnupfen.

Eine grosse Schwierigkeit besteht darin, dass die moderne ICD-Klassifikation keine Nosologie des Wirts und der Wirtskräfte kennt und in der Praxis keine vernünftige Prädiktivität für die Schwere und den Verlauf einer spezifischen Krankheitsdiagnose im Einzelfall besitzt [10]. Sie ist Ausdruck des limitierten und unsensitiven modernen medizinischen Wahrnehmens, und so werden mangelnde Widerstandskräfte mit der ICD-Klassifikation nicht erkannt. Dieser auf Spezifität getrimmten Nosologie fehlt eine ausreichende Sensitivität zum Erfassen wichtiger prognostischer Krankheitsmerkmale, vor allem wenn diese anamnestischer Natur sind [10, 11]. Diese Lücke lässt sich nicht schnell und einfach füllen. Sie führt aber dazu, dass wir bei der erfolgreichen Behandlung oder Verhinderung spezifischer Krankheitsäusserungen am Schluss nicht wissen, ob der Patient gesünder geworden oder nur belastet worden ist und ob er einen echten Nutzen hat.

\section{Wirksamkeit von Grippeimpfungen und das Wesen von Impfungen}

Weil Grippeviren im Gegensatz zu Pocken und Masern mutieren, hinterlassen sie keine generelle Grippeimmunität. Impfstoffantigene müssen den Mutationen angepasst werden, um überhaupt zu wirken, und deshalb hinterlassen Grippeimpfungen ebenfalls keine generelle Grippeimmunität. Theoretisch wird damit die Zielsicherheit und die mögliche Wirksamkeit von Impfungen gegen Grippe eher geschmälert; vervielfacht wird hingegen das potenzielle Verkaufsvolumen des ständig zu ändernden Impfstoffs.
Jefferson et al. [12] hatten 2006 in einer systematischen Analyse («systematic review») das Fehlen eines methodisch validen Nutzennachweises der Grippeimpfung festgestellt, denn in den vorhandenen Studien lassen sich zahlreiche mögliche «Biases» und Fehlerquellen aufzeigen. Eine weitere Studie der gleichen Gruppe unter der Ägide der Cochrane Collaboration zeigt, dass auch ein Wirkungsnachweis der Grippeimpfung bei Älteren fehlt [13], wenn man methodisch adäquate Massstäbe ansetzt. Theoretisch wäre die Grippeimpfung zwar bei den anfälligen älteren Menschen besonders von Nutzen, doch scheint gerade bei schwächeren älteren Menschen auch die Kraft für eine spezifische Immunantwort zu fehlen. Der Immunologe Jefferson hat für diese Analyse die Methoden der klinischen Epidemiologie verwendet.

Aus einer systematischen, klinisch-epidemiologischen Sicht und aufgrund klinischer Erfahrungen sorgfältig beobachtender Ärzte lassen sich die Wirkung von Viren und Impfungen wie auch das klinische Wesen von Impfungen mindestens hypothetisch als ein Glücksspiel einordnen: Ein Wirt mit einer guten Abwehr kommt ohne Impfungen gut durch und hat auch keine Impfnebenwirkungen $\mathrm{zu}$ befürchten. Ein Wirt mit einer prekären oder momentan geschwächten Abwehrkraft kann durch Viren, aber - in einem ebenso ungewissen Ausmass - auch durch Impfungen beeinträchtigt werden. Denn ein schon schwaches Abwehrsystem wird belastet, indem es durch das Impfen zu einer spezifischen Immunantwort genötigt wird, die es in der Regel nie benötigt. Das kompromittiert vermutlich den Schutz gegen konkurrierende Infektionen, oder es führt in seltenen Fällen sogar zu einem Impfschaden, wenn das Abwehrsystem «kippt» und die Impfnoxe eindringen kann. Es ist so gesehen ein Glücksspiel mit unbekannten Variablen, ob eine Impfung gerade vor etwas schützt, das wirklich eintreten würde, oder ob sie lediglich ein schon schwaches Abwehrsystem belastet und dadurch die unspezifische Krankheitsanfälligkeit in einem ungünstigen Moment erhöht. Da in der Regel Tausende geimpft werden müssen, um vielleicht einen einzigen spezifischen Komplikations- oder Todesfall zu verhüten, ist der Nutzen von Impfungen auf das ganze Morbiditäts- und Mortalitätsgeschehen von vornherein fraglich (zu hohe «number needed to treat» (NNT)).

Methodisch gute Studien, die einen Grippeimpfnutzen in kleinen Gruppen aufzeigen (junge schwangere Frauen in Bangladesch), scheinen zu bestätigen, dass ein hohes «Vorbehandlungsrisiko» mit einer entsprechend geringen NNT die Voraussetzung für einen möglichen Nutzen sind. In dieser Studie erkrankten über 90\% der Neugeborenen an einem fieberhaften Atemwegsinfekt, wobei die Kinder geimpfter Mütter nur in 34\% einen Arztbesuch benötigten im Vergleich zu 59\% der ungeimpften Gruppe - eine NNT von nur 6 [14]. Eine offene Frage bleibt, ob ein klinisch relevanter Nutzen von Dauer bestehen wird. Zudem fehlt 
solchen relativ kleinen - wenn auch an sich interessanten Einzelstudien eine ausreichende Repräsentativität.

\section{Schlussfolgerungen}

Eine systematische Betrachtung zeigt auf, dass der Blutzoll von Virusepidemien oder -pandemien nicht einfach vom Virus und seiner Variation abhängen, sondern möglicherweise nur vom gesundheitlichen Vorzustand des Wirts bzw. der Bevölkerung. Viren mutieren und können so überleben. Um zu überleben, dürfen sie den Wirt nicht zu stark dezimieren. Es ist eine blosse Theorie und wahrscheinlich eine Fehlinterpretation, dass in der Praxis Mutationen die Gefährlichkeit von Viren bestimmen. Nicht die spezifische Immunität, sondern «Mutationen» (bzw. Unterschiede) in der unspezifischen Immunbereitschaft des Wirts korrelieren mit einem komplizierten Ausgang von Virusinfektionen. Wenn die spezifische Immunität fast allein Gegenstand der heutigen medizinischen Scholastik ist, so scheint diese inzwischen weitgehend ohne reelle epidemiologische Relevanz zu sein. Klinische Epidemiologen oder Mathematiker bzw. Stochastiker wissen, dass eine Maximierung der Spezifität regelmässig auf Kosten der Sensitivität geht - auf Kosten der klinischen Relevanz und der Generalisierbarkeit [11]. Eine «unspezifische klinische Immunbereitschaft» lässt sich allerdings nicht einfach und leicht an im Labor gemessenen Antikörpern festmachen, sondern kann nur anhand klinisch-anamnestischer Daten abgeschätzt werden, die oft wenig eindeutig und wenig spezifisch sind $[10,11]$. Dafür ist eine solche klinische Einschätzung der Widerstandskräfte potenziell viel sensitiver und klinisch relevant.

Die Frage nach dem Nutzen wird noch weiter kompliziert durch die Möglichkeit, dass ein kompetenter Wirt durch die erfolgreiche Auseinandersetzung mit einer Grippe einen gesundheitlichen Gewinn haben könnte. Sogar eine gut nachgewiesene Senkung von Todesfällen oder Komplikationsfolgen durch eine spezifische Infektionskrankheit ist - sorgfältig betrachtet - ein zunächst nur vielleicht möglicher Nutzen, aber kein sicherer Nutzen. Am Schluss kommt es auf die Gesamtmorbidität und -mortalität an. Kontrollierte Studien zu diesen entscheidenden Fragen sind schwierig und haben bei Impfungen schon immer gefehlt. Die vorhandene Evidenz jenseits von Wunschtheorien weist aber darauf hin, dass auch «erfolgreiche» Impfungen letztlich nutzlos geblieben sind und die Gesamtsterblichkeit nie senken konnten - es wurde lediglich die spezifische Mortalitätsursache ausgetauscht.
Wir brauchen das epidemiologische Mass der Gesamtsterblichkeit - oder allenfalls das Total aller relevanten klinischen Endpunkte. Und deshalb würden sogar methodisch stichhaltige Ergebnisse über Verbesserungen von Grippestatistiken (die bis heute fehlen) nicht viel bedeuten. Das jeweilige Fortschrittsloblied auf methodisch nicht stichhaltige Studienergebnisse und die scheinbar unvermeidliche Vermarktung scheinbarer Erfolge zeigen nur das Ausmass von Wunschdenken oder Geschäftssinn, sind aber keine seriöse Medizin und Wissenschaft. Man kann leicht erkennen, dass der breite Konsensus unter Immunologen und Ärzten über den Einsatz von Grippeimpfungen und über das Vorgehen gegen die angebliche Schweinegrippe-Pandemie weder durch gute empirische Evidenz gestützt noch theoretisch sorgfältig begründet ist.

Dass die Schweinegrippe nie gefährlich war und Warnungen mit der - nur scheinbaren - Gefährlichkeit von Mutationen den Aktivismus begründen konnte, ist nur ein Beispiel. Es weist auf die generelle Tatsache hin, dass die moderne «Medical Community» die Bedeutung des Wirts und seiner (unspezifischen) Widerstands- und Gesunderhaltungskräfte vergessen und so das Augenmass verloren hat. Wir glauben, dass die falschen Annahmen und Schlussfolgerungen der WHO zur Schweinegrippe-Pandemie nicht durch blanke Korruption entstanden sind, sondern durch kollektives Wunschdenken und nachlässige Wissenschaft - durch scholastische Analysen ohne Augenmass. Unsere Studie stellt nicht das Impfen insgesamt infrage, aber der Nutzen und die Bedeutung von Impfungen wird sicherlich stark relativiert und auf kleine Gruppen, wie möglicherweise Schwangere in Dritte-Welt-Ländern, begrenzt. Es ist interessant, darauf hinzuweisen, dass der Wirtszustand auch bei anderen Krankheiten - z.B. Krebs, Rheuma, Multiple Sklerose usw. - die entscheidende und wissenschaftlich vernachlässigte Determinante von Krankheitsverläufen und der Mortalität sein könnte und nicht die scheinbare Aggressivität oder Bösartigkeit der jeweiligen Krankheit [15].

\section{Dank}

Diese Arbeit ist dem Polnischen Gesundheitsministerium gewidmet. Am 25. Mai 2010 wurde diese Studie Vize-Gesundheitsminister Adam Fronczak im Polnischen Gesundheitsministerium überreicht.

\section{Disclosure Statement}

The authors did not receive any kind of support for this article. A conflict of interest does not exist. 


\section{Literatur}

1 Watson R: WHO is accused of 'crying wolf' over swine flu pandemic. BMJ 2010;340:c1904

2 Kloth HM: Grippe-Katastrophe von 1918/19. Zeitgeschichten auf Spiegel-Online, 27.04.2009. http://einestages.spiegel.de/static/topicalbum background/4024/_nehmen_sie_alle_tischler_ und_lassen_sie_saerge_herstellen.html.

3 Reid AH, Janczewski TA, Lourens RM, Elliot AJ, Daniels RS, Berry CL, Oxford JS, Taubenberger JK: 1918 influenza pandemic caused by highly conserved viruses with two receptor-binding variants. Emerg Infect Dis 2003;9:1249-1253.

4 Feinstein AR: Why do we need clinical epidemiology? - A practice-oriented clinical science; in Schmidt JG, Steele RE (Hrsg): Kritik der medizinischen Vernunft: Schritte zu einer zeitgemässen Medizin - ein Lesebuch. Mainz, Kirchheim, 1994, pp 233-243.

5 Schmidt JG: Die Vision einer pragmatischen klinischen Forschung oder das Ende der Diskussion über «Placebo» und «spezifische Wirkungen»; in Schmidt JG (Hrsg): Placebo - wertvoll wenn es dem Patienten nützt? Methodologie einer nutzenorientierten, pragmatischen klinischen Forschung. Forsch Komplementärmed 1998;5(suppl 1):102-111.
6 Mayr A: Diskussion zu dem Beitrag Eradikation und Tilgung von Seuchen. Dtsch Arztebl 2007;104:1248.

7 Colgrove J: The McKeown thesis: a historical controversy and its enduring influence. Am J Public Health 2002;92:725-729.

8 Doshi P: Trends in recorded influenza mortality: United States, 1900-2004. Am J Public Health 2008;98:939-945.

9 Pandemic (H1N1) 2009 - update 88. www. who.int/csr/don/2010_02_19/en/index.html.

10 Feinstein AR: The hard data creed in current clinical practice: its spurious validity and the challenge to define meaningful clinical variables; in Schmidt JG, Steele RE (Hrsg): Kritik der medizinischen Vernunft: Schritte zu einer zeitgemässen Medizin - ein Lesebuch. Mainz, Kirchheim, 1994, pp 210-218.

11 Schmidt JG (ed): Placebo - wertvoll wenn es dem Patienten nützt? Methodologie einer nutzenorientierten, pragmatischen klinischen Forschung. Forsch Komplementärmed 1998;5(suppl 1).

12 Jefferson $\mathrm{T}$ : Influenza vaccination: policy versus evidence. BMJ 2006;28:912-915.
13 Rivetti D, Jefferson T, Thomas R, Rudin M, Rivetti A, Di Pietrantonj C, Demicheli $\mathrm{V}$ : Vaccines for preventing influenza in the elderly. Cochrane Database Syst Rev 2006;3:CD004876.

14 Zaman K, Roy E, Arifeen SE, Rahman M, Raqib R, Wilson E, Omer SB, Shahid NS, Breiman RF, Steinhoff MC: Effectiveness of maternal influenza immunization in mothers and infants. N Engl J Med 2008;359:15551564

15 Schmidt JG: Ist Verzicht immer Nihilismus? Über das Handeln aufgrund rationaler Erkenntnisse; in Köbberlin J (Hrsg): Zeitfragen der Medizin. Berlin, Springer, 1997, pp 115125 . 